\title{
FORMACION DE PALYGORSKITA ASOCIADA AL FLUJO REGIONAL DE LAS AGUAS SUBTERRANEAS DEL BORDE S.O. DE LA CUENCA DEL DUERO (*)
}

\author{
F. J. Sánchez San Román $\left({ }^{* *}\right)$ y J. A. Blanco Sánchez (**)
}

\begin{abstract}
RESUMEN.
En este trabajo se presentan algunos datos sobre la geoquímica de las aguas subterráneas del paleógeno del S.O. de la Cuenca del Duero. Estos datos muentran una clara disminución de las concentraciones de sílice y de $\mathrm{Mg}$ a lo largo del flujo regional desde las áreas de recarga hacia las áreas de descarga. Por otra parte, en una de la formaciones paleógenas de la zona -Areniscas de Aldearrubia - hemos detectado la presencia de pequeñas cantidades de palygorskita. Dicho mineral se presenta en cristales perfectamente automorfos que tienden primero a tapizar y después a cerrar la porosidad.

La posibilidad, demostrada termodinámicamente, de tranformación de esmectitas - muy abundantes en la citada formación - en palygorskita mediante aporte de sílice y Mg a partir del agua subterránea satisface todos los datos analíticos y de observación. Este tipo de génesis de la palygorskita asociado al flujo actual o subactual del agua subterránea puede considerarse como una variante del origen diagenético.
\end{abstract}

Palabras clave: Cuencas del Duero, paleógeno, agua subterránea, silice, magnesio, palygorskita, arenisca, porosidad, diagénesis.

\begin{abstract}
The present work provides some data concerning the geochemistry of the paleogene groundwater in the S.W. region of the Duero Basin. The data reflect a clear decrease in silica and $\mathbf{M g}$ throughout the regional flow from recharge areas to discharge ones. In one of the Paleogenes Formations of the area -Areniscas de Aldearrubia - the presence of small amounts of palygorskite was detected. This mineral appears in perfectly automorphic crystals with a tendency to envelope and then close porosity.

From thermodinamic criteria, the possibility of the conversion of smectite -very abundant in the Formation - into palygorskite by addition of graundwater silica and $\mathrm{Mg}$ is in accordance with all the analytical and observational data. The palygorskite genesis related to present or past groundwater flow could be considered as a variant of the diagenetic origin for this mineral.
\end{abstract}

Key words: Dueron basin, paleogene, groundwater, silica, magnesium, palygorskite, sandstone, porosity, diagnenesis.

\section{Introducción}

La composición química del agua subterránea varía a lo largo de su recorrido de acuerdo con diversos procesos bien conocidos: disolución, precipitación, intercambio catiónico, etc. En formaciones con una proporción considerable de arcillas pueden presentar una cierta importancia los procesos de transformación y neoformación de minerales arcillosos, en los que intervienen directamente los elementos disueltos en las aguas subterráneas. La idea no es nueva, y ya Glass, et al. (1956) y Potter y Glass (1958) pusieron de manifiesto diferencias significativas en las proporciones de minerales arcillosos en áreas o niveles con mayor permeabilidad, es decir, con un flujo de agua subterránea más activo. No obstante, en la bibliografia más reciente no es frecuente que se invoquen estos procesos para explicar las variaciones de la composición

(*) Trabajo parcialmente subvencionado por los proyectos $1785 / 82$ y $1113 / 84$ de la CAYCIT.

(**) Dpto. Geomorfología y Geotectónica. Fac. Ciencias. Universidad de Salamanca. 37008 Salamanca. 
química del agua subterránea a lo largo del flujo regional.

En este trabajo presentamos algunos datos sobre la geoquímica de las aguas subterráneas del paleógeno del borde SO de la Cuenca del Duero. En dicha zona hemos observado que las concentraciones de sílice y de $\mathrm{Mg}^{++}$disminuyen desde las áreas de recarga hacia las áreas de descarga del flujo regional.

Por otra parte, en una de las formaciones paleógena de la zona - Areniscas de Aldearrubia - hemos detectado una neoformación de palygorskita asociada a la porosidad de estos materiales que no encaja en ninguno de los modelos de génesis propuestos para este mineral. En efecto, en la síntesis sobre los depósitos de palygorskita y sepiolita de los materiales terciarios españoles de Galán y Castillo (1984) la génesis de la palygorskita aparece ligada a precipitación directa o diagénesis en medios alcalinos ricos en sílice y Mg. Otros trabajos referidos más concretamente a la palygorskita o sepiolita de la Cuenca del Duero proponen ambién mecanismos de formación asociados sea a la etapa puramente sedimentaria en los paleosuelos (Corrochano et al., 1986), sea a la etapa de diagénesis precoz (Leguey et al., 1984; Armenteros et al., 1986).

En este trabajo aportamos diversas evidencias que traten de probar que las dos observaciones citadas, perdida de silice y $\mathrm{Mg}^{++}$a lo largo del flujo regional del agua subterránea y neoformación de palygorskita asociada a la porosidad, están estrechamente ligadas.

\section{Area de estudio}

El área en que se ha estudiado la evolución geoquímica de las aguas subterráneas corresponde al paleógeno que aflora al SO de la Cuenca del Duero, limitada al N por el río Duero y al E por la divisoria hidrográfica entre los ríos Guareña y Trabancos (fig. 1).

La geología es compleja, y resulta difícil correlacionar las facies definidas por los diversos autores que han estudiado la zona (Jiménez, 1972, 1973; Corrochano, 1977, 1982; I.G.M.E., 1980a, 1980b; Alonso Gavilán, 1981). En general, se trata de depósitos detríticos de origen continental, con predominancia de finos (fangos, areniscas con abundante matriz fangosa) e intercalaciones de arenas y gravas.

El conjunto, todo el paleógeno de la zona se considera dividido en tres tramos o unidades. La mineralogía de arcillas ha sido estudiada en la formación Areniscas de Aldearrubia, que forma parte de la unidad superior, y que aflora al SE de la zona (fig. 1).

Una descripción detallada de los principales afloramientos de esta formación, así como de sus características estratigráficas y sedimentológicas puede encontrarse en Alonso Gavilán (1981 y 1984), Alonso

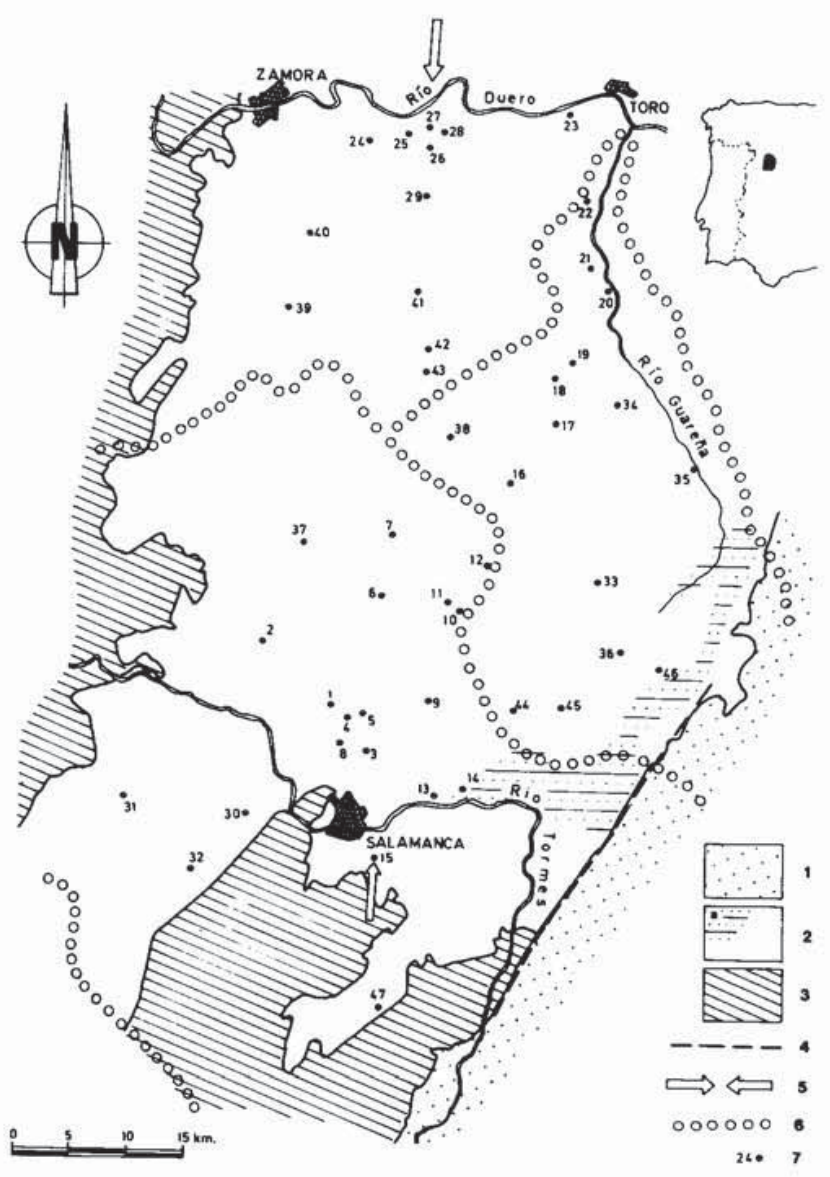

Fig. 1.-Zona de estudio. 1. Mioceno.-2. Paleógeno con recubrimientos someros miocenos y cuaternarios.-2a. Principales afloramientos de la formación Areniscas de Aldearrubia.-3. Zócalo hercínico.-4. Falla Alba-Villoria.-5. Situación del corte de la figura 3.-6. Divisoria hidrográfica.-7. Sondeo muestreado y número de referencia.

Gavilán y Armenteros (1983) y Alonso Gavilán et al. (1986). Esta formación de origen fluvial está organizada en ciclos semejantes al representado en la figura 2 siendo sus facies más características las siguientes:

- Arenas con rubefacción más o menos intensa pero generalizada y alta porosidad debida al buen calibrado y a la casi ausencia de matriz arcillosa; se interpretan asociadas a depósitos de canal.

- Arcillas verdes en capitas muy homogéneas salvo que estén afectadas por procesos de escape de fluidos o bioturbación; se interpretan como depósitos de relleno de canales abandonados en llanuras de inundación próximas a canales activos.

- Fangos ocres bioturbados de aspecto muy compacto y disyunción en bolos con procesos frecuentes, pero no intensos, de encostramiento calcáreo; se interpretan como depósitos de llanura de inundación, alejados de los canales activos, colonizados por paleosuelos. 


\section{Flujo del agua subterránea en el área de estudio}

El flujo del agua subterránea es complejo y difícil de conocer con precisión al no existir una red piezométrica de calidad. Se trata de un sistema de flujo tridimensional, de acuerdo con el esquema clásico de Hubert (1940), con superposición de flujos locales, intermedios y regionales (Toth, 1963) que no pueden definirse correctamente por la carencia de datos piezométricos precisos.

Una idea general del flujo ha sido expuesta por Martí et al. (1979) e I.G.M.E. (1980 c, 1982). En la figura 3 presentamos un modelo simplificado de dicho flujo. Los ríos Duero y Tormes actúan como líneas de drenaje más importantes, y diversos arroyos generan sistemas de flujo locales, en ocasiones oblícuos o perpendiculares a la dirección general del flujo N-S mostrada en la citada figura 2. Al este del corte representado en dicha figura el modelo se complica ligeramente y la divisoria superficial entre el Duero y el Tormes no constituye ya divisoria de las aguas subterráneas, sino que en profundidad todo el flujo es hacia el Duero (Martí et al., op. cit.; I.G.M.E., op. cit.).

\section{Geoquímica de las aguas subterráneas}

\section{Generalidades}

Se han analizado 47 muestras de aguas subterráneas procedentes de otros tantos sondeos distribuidos por toda la zona, y cuyas profundidades oscilan en la mayoría de los casos entre 80 y 160 metros. No es posible precisar la profundidad del nivel o niveles acuíferos de donde proceden las muestras, pues la entubación está ranurada en toda su longitud, y las muestras fueron tomadas por bombeo.

Se midieron en el campo conductividad, temperatura y $\mathrm{pH}$, y en laboratorio los iones mayoritarios y la silice. Los cationes y la sílice se analizaron por absorción atómica. En la figura 1, se presenta la situación de los sondeos muestreados y en la Tabla 1 se incluyen los datos de interés para la exposición subsiguiente.

En general, se trata de aguas poco salinas, cuyas conductividades oscilan entre 200 y $600 \mu \mathrm{mhos} / \mathrm{cm}$., a excepción de algunos sondeos próximos al río Duero, que captan aguas con conductividades de 3.000 a $4.000 \mu \mathrm{mhos} / \mathrm{cm}$. En su mayoría son bicarbonatadas cálcico-magnésicas, tendiendo a sódicas en las áreas de descarga. Las aguas salobres citadas más arriba son clorurado-sulfatadas sódicas (Sánchez, 1983).

\section{Concentraciones de sillice y magnesio y su relación con el flujo}

Las concentraciones de sílice de los sondeos citados oscilan entre 10,1 y $41,9 \mathrm{ppm}$. de $\mathrm{SiO}_{2}$, con un valor

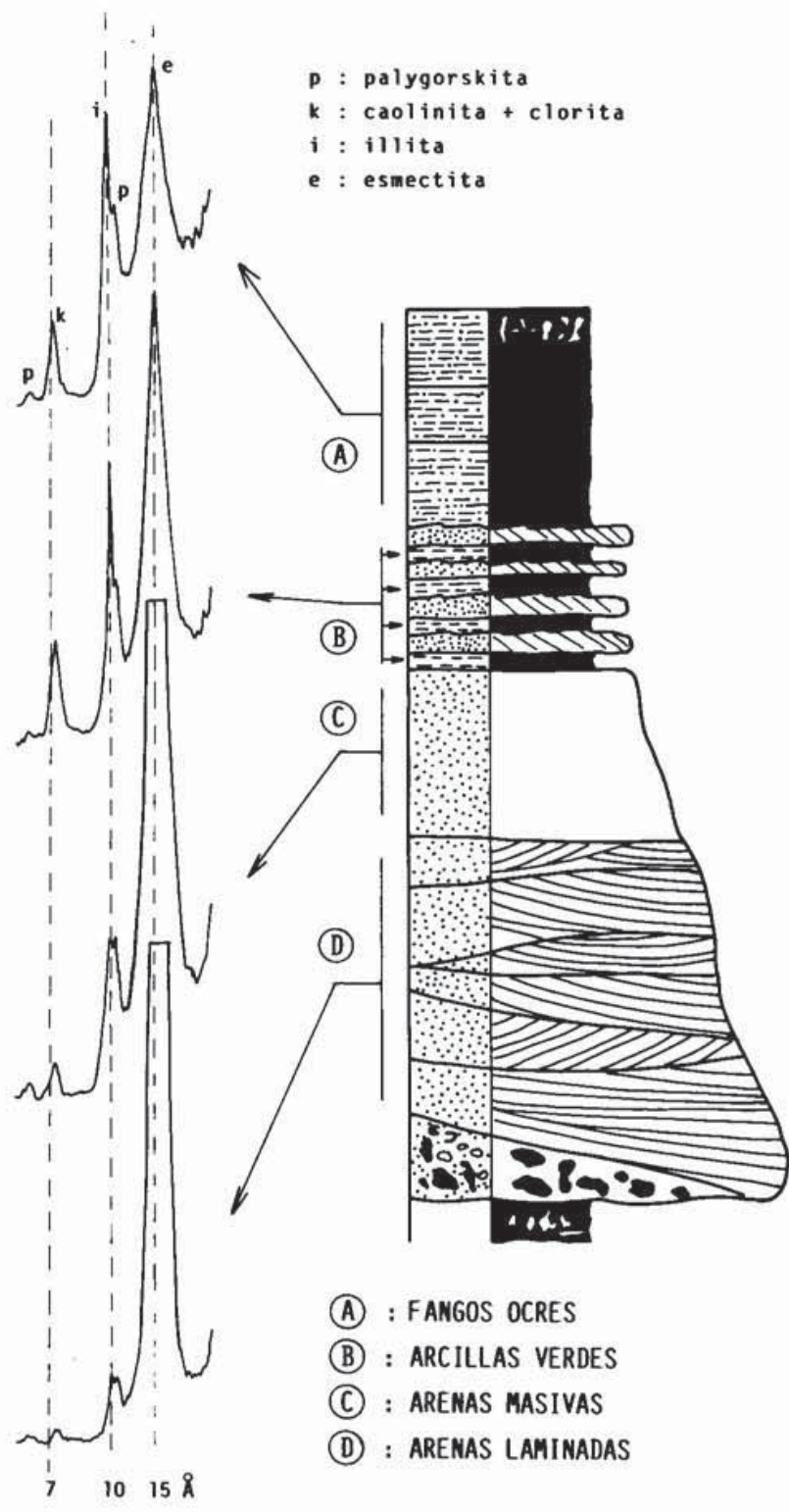

Fig. 2.-Ciclo ideal mostrando las diversas facies de la formación areniscas de Aldearrubia y difractogramas de la fracción inferior a

$2 \mu$ (ligeramente modificado a partir de Alonso et al., 1986).

medio de 25,6 ppm. Los valores mínimos se registran en las áreas de descarga del flujo regional, y resulta evidente su disminución a lo largo de dicho flujo (datos en ppm. de $\mathrm{SiO}_{2}$ ):

\begin{tabular}{|c|c|c|c|c|c|}
\hline & & Recarga & Transición & Descarga & Total \\
\hline & & 41 , & 42,6 & 21,5 & 41,9 \\
\hline Media & & 32,4 & 24,8 & 16,4 & 25,6 \\
\hline Mínimo & $\ldots$. & 21,9 & 13,2 & 10,1 & 10,1 \\
\hline
\end{tabular}


T A B L A $n=1$

DATOS HIDROQUIMICOS

\begin{tabular}{|c|c|c|c|c|c|c|}
\hline \multirow{2}{*}{\multicolumn{2}{|c|}{$\stackrel{\text { No }}{\text { Ref. }}(*)$}} & \multirow[b]{2}{*}{$\mathrm{pH}$} & \multicolumn{2}{|c|}{ Magnesio } & \multicolumn{2}{|c|}{ Silice } \\
\hline & & & $\begin{array}{l}\text { Conc. } \\
\text { (ppm) }\end{array}$ & $\begin{array}{l}\text { Activ. } \\
\text { (mol/l.) }\end{array}$ & $\begin{array}{l}\text { Conc. } \\
(\mathrm{ppm} \\
\left.\mathrm{SiO}_{2}\right) \\
\end{array}$ & $\begin{array}{l}\text { Activ. } \\
\text { (mol/1.) }\end{array}$ \\
\hline 1 & D & 8.3 & 21.9 & $6.46 E-04$ & 20.7 & 3. $45 E-04$ \\
\hline 2 & $T$ & 8.3 & 16.1 & 4. $95 \mathrm{E}-04$ & 27.6 & $4.6 E-04$ \\
\hline 3 & $T$ & 8.3 & 46.9 & 1.33E-03 & 21.3 & $3.55 E-04$ \\
\hline 4 & D & 8.8 & 4.6 & 1. $37 E-04$ & 15.7 & $2.62 E-04$ \\
\hline 5 & $D$ & 8.9 & 3.4 & $9.92 E-05$ & 12.6 & 2. $1 \mathrm{E}-04$ \\
\hline 6 & $R$ & 8 & 34.2 & $1 E-03$ & 28.2 & 4. 7E-04 \\
\hline 7 & $\mathrm{R}$ & 8.2 & 23.7 & $6.97 E-04$ & 34.4 & $5.73 E-04$ \\
\hline 8 & D & 8.1 & 42.4 & $1.22 \mathrm{E}-03$ & 20.7 & 3. $45 E-04$ \\
\hline 9 & $\mathrm{R}$ & 8.1 & 54.5 & $1.53 E-03$ & 23.1 & $3.85 E-04$ \\
\hline 10 & 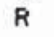 & 8 & 27.2 & $7.98 \mathrm{E}-04$ & 41.9 & $6.98 E-04$ \\
\hline 11 & $\mathbf{R}$ & 7.9 & 27.2 & $7.97 E-04$ & 38.8 & $6.47 E-04$ \\
\hline 12 & R & 8.3 & 18 & $5.37 E-04$ & 34.4 & 5. $73 E-04$ \\
\hline 13 & $\mathrm{R}$ & 8.4 & 21.4 & 6. $17 E-04$ & 22.6 & $3.77 E-04$ \\
\hline 14 & $\mathbf{R}$ & 8.1 & 28.2 & 8. $23 \mathrm{E}-04$ & 37.6 & $6.27 E-04$ \\
\hline 15 & $\mathrm{R}$ & 8.4 & 23.1 & $6.64 E-04$ & 36.3 & $6.05 E-04$ \\
\hline 16 & $T$ & 8.2 & 14.1 & 4.33E-04 & 14.4 & 2. $4 \mathrm{E}-04$ \\
\hline 17 & $\begin{array}{ll}T \\
T\end{array}$ & 8.3 & 17.3 & $5.23 E-04$ & 20.7 & $3.45 E-04$ \\
\hline 18 & T. & 8 & 24.4 & $7.29 E-04$ & 21.3 & $3.55 E-04$ \\
\hline 19 & $T$ & 8 & 18.5 & 5.55E-04 & 20.1 & $3.35 E-04$ \\
\hline 20 & $T$ & 7.9 & 31.6 & $9.27 E-04$ & 24.4 & $4.07 E-04$ \\
\hline 21 & $T$ & 7.8 & 27.6 & $7.9 E-04$ & 21.9 & $3.65 E-04$ \\
\hline 22 & D & 8.2 & 10.7 & $3.38 \mathrm{E}-04$ & 15.7 & $2.62 \mathrm{E}-04$ \\
\hline 23 & D & 8.6 & 2.6 & $8.08 E-05$ & 21.9 & $3.65 E-04$ \\
\hline 24 & D & 8.2 & 31.7 & 8. $47 E-04$ & 21.9 & $3.65 E-04$ \\
\hline 25 & D & 8.1 & 6.4 & $1.71 \mathrm{E}-04$ & 10.1 & $1.68 \mathrm{E}-04$ \\
\hline 26 & $T$ & 8.2 & 28 & 8. $11 \mathrm{E}-04$ & 23.8 & $3.97 E-04$ \\
\hline 27 & D & 8.2 & 16.3 & $3.94 E-04$ & 13.8 & 2. 3E- 04 \\
\hline 28 & D & 8.2 & 11.7 & 2. $85 \mathrm{E}-04$ & 11.9 & $1.98 \mathrm{E}-04$ \\
\hline 29 & $T$ & 7.7 & 13.4 & 4. $3 E-04$ & 20.7 & 3. $45 E-04$ \\
\hline 30 & $T$ & 7.8 & 29.9 & $8.68 E-04$ & 27.6 & $4.6 E-04$ \\
\hline 31 & 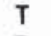 & 8.3 & 13 & $3.77 E-04$ & 13.2 & 2. $2 \mathrm{E}-\mathrm{O} 4$ \\
\hline 32 & $\begin{array}{l}T \\
\end{array}$ & 7.8 & 33.3 & $9.62 E-04$ & 36.3 & $6.05 E-04$ \\
\hline 33 & $T$ & 7.8 & 27.6 & $8.09 E-04$ & 30.7 & $5.12 E-04$ \\
\hline 34 & $T$ & 8 & 21.4 & $6.39 E-04$ & 21.3 & $3.55 E-04$ \\
\hline 35 & D & 8 & 4.9 & $1.56 E-04$ & 15.7 & $2.62 E-04$ \\
\hline 36 & T & 7.9 & 16.9 & $5.02 E-04$ & 42.6 & $1 E-04$ \\
\hline 37 & R & 7.8 & 17.3 & $5.41 E-04$ & 27.6 & 4. $6 E-04$ \\
\hline 38 & R & 8 & 26.3 & $7.7 E-04$ & 33.2 & $5.53 E-04$ \\
\hline 39 & $\mathbf{R}$ & 7.8 & 40.9 & 1. $14 \mathrm{E}-03$ & 28.2 & 4. $7 E-04$ \\
\hline 40 & $T$ & 8 & 25.2 & $7.48 E-04$ & 18.2 & $3.03 E-04$ \\
\hline 41 & $T$ & 7.6 & 23.8 & $6.81 E-04$ & 36.3 & $6.05 E-04$ \\
\hline 42 & $R$ & 8.1 & 27.1 & 8. $1 E-04$ & 21.9 & $3.65 \mathrm{E}-04$ \\
\hline 43 & $\mathrm{R}$ & 8.1 & 31.3 & $9.1 E-04$ & 29.4 & 4. $9 E-04$ \\
\hline 44 & $\mathrm{R}$ & 7.8 & 15.8 & $4.65 E-04$ & 37.6 & $6.27 \mathrm{E}-04$ \\
\hline 45 & $\mathrm{R}$ & 8.1 & 11.6 & $3.52 E-04$ & 38.2 & $6.37 E-04$ \\
\hline 46 & $T$ & 8.1 & 13 & $3.88 E-04$ & 29.4 & $4.9 E-04$ \\
\hline 47 & $\mathrm{R}$ & 7.8 & 25.4 & $7.34 E-04$ & 36.9 & $6.15 E-04$ \\
\hline
\end{tabular}

(*) Area de recarga, transiciōn o descarga
En el perfil de la figura 3 se muestra dicha evolución. Como puede observarse, la disminución referida es una tendencia general, apreciable estadísticamente, pero con diversas excepciones, debidas a variaciones en la litología o a la diferente profundidad de los niveles acuíferos explotados por los sondeos.

Las concentraciones de $\mathrm{Mg}^{++}$oscilan entre 2,6 y $54,5 \mathrm{ppm}$. con una media de 22,4 ppm. Análogamente a la sílice, los valores más elevados se presentan en áreas de recarga y los menores en áreas de descarga (datos en ppm. de $\mathrm{Mg}^{++}$):

\begin{tabular}{|c|c|c|c|c|c|}
\hline & & Recarga & Transició & Descarga & Total \\
\hline Máximo & & 54,5 & 46,9 & 42,4 & 54,5 \\
\hline Media & 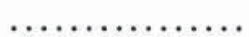 & 26,7 & 23,1 & 14,2 & 22,4 \\
\hline Mínimo & $\ldots \ldots \ldots \ldots \ldots$ & 11,6 & 13,0 & 2,6 & 2,6 \\
\hline
\end{tabular}

En la figura 4 se representa la relación entre las actividades de $\mathrm{Mg}^{++}$y de sílice para mostrar la disminución simultánea de ambos en el sentido del flujo del agua subterránea.

\section{Discusión de los datos hidroquímicos}

La silice y el $\mathrm{Mg}$ de las aguas subterráneas provienen de la alteración de los silicatos. Estos procesos son especialmente importantes en los suelos por las condiciones de acidez reinantes, pues las reacciones de disolución de los silicatos, a excepción de las formas de sílice pura, consumen $\mathrm{H}^{+} \mathrm{y}$, por tanto, su solubilidad se ve fvorecida en medio ácido. A partir del agotamiento de la reserva ácida del agua por pérdida del $\mathrm{CO}_{2}$ disuelto, las reacciones de disolución de los silicatos se amortiguan, porque el $\mathrm{pH}$ se eleva rápidamente. Este aumento del $\mathrm{pH}$ unido a la concentración iónica alcanzada motiva no sólo el cese de la disolución de algunos silicatos "primarios" (cuarzo, feldespato $\mathrm{K}$ ) por alcanzarse la saturación, sino que además provoca la precipitación de silicatos secundarios (caolinita y montmorillonita). Estos tres mecanismos (dislución de silicatos, saturación, neoformación de arcillas) regulan la concentración de sílice disuelta en el agua.

Todas estas reacciones han sido cuantificadas por Fritz (1975) mediante un complejo modelo de ordenador que simula la disolución de un granito en un perfil de alteración, por encima del nivel de saturación. Este autor demuestra como a partir de una agua prácticamente destilada (agua de lluvia) todos los minerales del granito se disuelven hasta que el agua alcanza $6 \mathrm{ppm}$. de silice disuelta; en ese momento cesa la disolución del cuarzo del granito pero la concentración en silice del agua sigue aumentando aportada por la disolución de los otros silicatos. A 40 


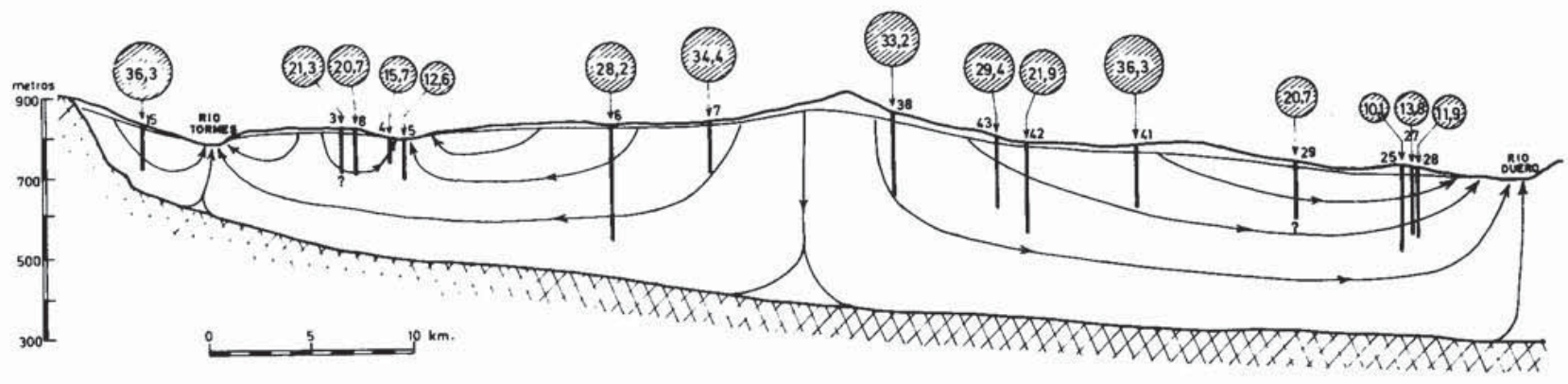

Fig. 3.-Perfil esquemático del flujo subterráneo, con indicación de las concentraciones de sllice en ppm.

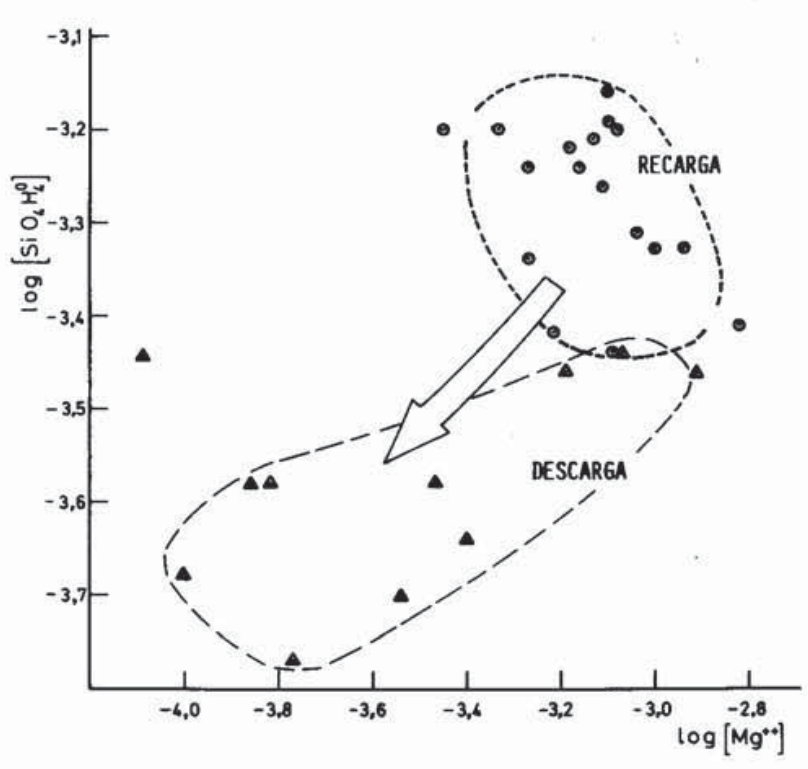

Fig. 4.-Relación entre las actividades de sílice y las de $\mathrm{Mg}$, mostrando su disminución simultánea hacia las áreas de descarga.

ppm. de silice disuelta, aproximadamente, siendo ya el $\mathrm{pH}$ del agua ligeramente alcalino se estabiliza la concentración de silice controlada por dos factores: aporte por disolución de silicatos que aún no han alcanzado la saturación (biotita, plagioclasa), y sustracción por neoformación de arcillas (primero caolinita y después esmectitas).

El comportamiento del $\mathbf{M g}^{++}$en el agua es similar al de la sílice, es decir, por una parte aumenta por disolución de silicatos ferromagnesianos, que son, en general, muy solubles, pero por otra parte es retirado del agua por precipitación, primero de esmectitas y después de carbonatos. En el modelo citado de Fritz, el máximo de $\mathrm{Mg}^{++}$en el agua de alteración se alcanza posteriormente a la estabilización de la sílice, es decir, cuando la reacción de disolución del granito está más avanzada y, por tantro, el pH es algo más básico.

De acuerdo con estas consideraciones resulta senci- llo comprender que en las áreas de recarga las concentraciones en sílice y en $\mathrm{Mg}^{++}$sean altas, pues son aguas que a lo largo de su infiltración por el perfil de alteración y por la zona no saturada han disuelto silicatos en función de su reserva ácida.

Muchos menos evidente aparece la disminución en las concentraciones asociada al flujo, que hemos descrito en el apartado anterior. Para la sílice, esta disminución ya ha sido atribuida a la neoformación de arcillas (Sánchez, 1982, 1983; Fernández y Llamas, 1983), aunque como una mera hipótesis y sin asociarla a la variación de otros componentes del agua subterránea. Las variaciones del $\mathbf{M g}^{++}$han sido clásicamente explicadas por intercambio catiónico o por precipitación de carbonatos, sin que ninguno de estos procesos haya sido claramente detallado; en cualquier caso, no conocemos ningún intento de correlacionar sus variaciones con las de la silice.

Un modelo conceptual de evolución que permite tratar conjuntamente las anomalías de la sílice y del $\mathrm{Mg}^{++}$es el establecido por Trauth (1977). Este autor expone diversas secuencias arcillosas observadas en varias cuencas sedimentarias desde el borde al centro y que se resumen así:

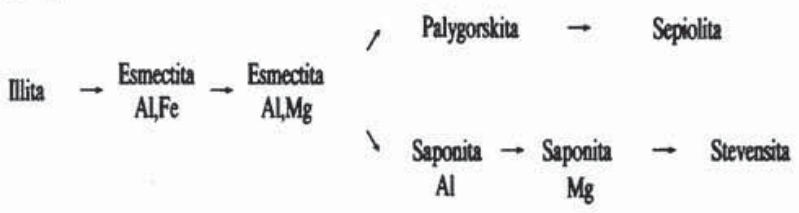

Este modelo necesita una incorporación progresiva de $\mathrm{Si}$ y de $\mathrm{Mg}$ desde la illita hasta cualquiera de sus dos polos finales. Según este autor en los primeros pasos (esmectitas) se producen solamente transformaciones; después (palygorskita, saponita) tienen lugar recristalizaciones sobre el soporte arcilloso existente, con cambios en la estructura y aumento en la cantidad de arcilla, y en los últimos pasos (sepiolita, stevensita) se producen realmente neoformaciones. En la bifurcación de la secuencia las dos vías son excluyentes: si la relación $\mathrm{Si} / \mathrm{Mg}$ es alta se forman minerales fibrosos (palygorskita, sepiolita), y si es baja, mincrales laminares (saponitas, stevensita). 
Trauth propuso el modelo anterior para explicar las variaciones arcillosas que observó en cuencas sedimentarias desde los bordes hacia el centro, atribuyéndoles un carácter sinsedimentario. Extrapolar este modelo al flujo regional del agua subterránea, asimilando borde de cuenca - zona de recarga, centro de cuencazona de descarga parece en primera aproximación atrevido. A pesar de ello vamos a intentar correlacionar la pérdida de sílice y de $\mathrm{Mg}^{++}$a lo largo del flujo subterráneo con neoformaciones anómalas de palygorskita que serán descritas en el apartado siguiente. Para ello hemos elegido el paso de la secuencia de Trauth esmectita-palygorskita -aunque termodinámicamente, y teniendo en cuenta la composición química de las aguas subterráneas de esta zona, otras transformaciones son posibles, lo que puede ponerse de manifiesto mediante la utilización de diversos diagramas de estabilidad (Tardy, 1969, 1982). En particular, el equilibrio entre la montmorillonita y la palygorskita puede formularse así (Weaver y Beck, 1977, pp. 190-192):

$5 \mathrm{PAL}+15,6 \mathrm{H}^{+}+24,4 \mathrm{H}_{2} \mathrm{O}=3 \mathrm{MONT}+7,65 \mathrm{Mg}^{++}+0,3 / \mathrm{n} \mathrm{X}^{\mathrm{n}+}+15,6 \mathrm{SiO}_{4} \mathrm{H}_{4}$ (1)

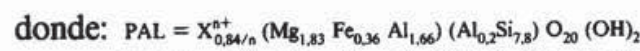

MONT $=\mathrm{X}_{0,7 / n}^{+}+\left(\mathrm{Mg}_{0,5} \mathrm{Fe}_{0,0} \mathrm{Al}_{2,9}\right)\left(\mathrm{Al}_{0,2} \mathrm{Si}_{7,8}\right) \mathrm{O}_{20}(\mathrm{OH})_{4}$ $\mathrm{X}^{\mathrm{n+}}=\mathrm{Na}^{+}, \mathrm{Ca}^{\mathrm{a}}, \mathrm{Mg}^{++}$.

El diagrama de estabilidad deducido de esta reacción es tridimensional (Weaver y Beck, op. cit.). de difícil visualización, por lo que consideraremos el problema numéricamente.

La constante de equilibrio de la reacción (1), considerando $\mathrm{X}=\mathrm{Mg}$, sería:

$$
\mathbf{K}_{\text {PALMONT }}=\frac{\left|\mathrm{Mg}^{++}\right| 7,80\left|\mathrm{SiO}_{4} \mathrm{H}_{4}\right|^{15,6}}{\left|\mathbf{H}^{+}\right|^{15,6}}
$$

o bien:

$\log \mathrm{K}_{\text {PALMONT }}=7,80\left(\log \left|\mathrm{Mg}^{++1}\right|+2\left|\mathrm{SiO}_{4} \mathrm{H}_{d}\right|+2 \mathrm{pH}\right)(3)$.

Estos autores calculan que $\log \mathrm{K}_{\mathrm{PAL}-\mathrm{MONT}}=44,85$. Aplicando las fórmulas (2) o (3), con los valores de una solución concreta se puede comprobar si la reacción (1) se desarrollará en uno u otro sentido. $\mathrm{Si}$ denominamos Producto de Actividades Iónicas (PAI) al resultado de la expresión (2) en dicho caso, el grado de equilibrio vendrá expresado por el índice $\mathrm{PAI} / \mathrm{K}$, o bien por $\log (\mathrm{PAI} / \mathrm{K})$, que serán, respectivamente, 1 y 0 en el equilibrio.

Aplicando la expresión (3) con los datos analíticos que figuran en la Tabla 1, se obtiene que en el $80 \%$ de los casos log (PAI/K) es mayor que 0 (log PAI $>44,85$ ) lo que indica que corresponden a aguas que no están en equilibrio con la montmorillonita y la palygorskita, sino que la reacción (1) se produce hacia la izquierda, es decir, transformación de montmorillonita en palygorskita.
Los valores del índice $\log (\mathrm{PAI} / \mathrm{K})$ muestran una clara relación con el flujo regional del agua subterránea:

\begin{tabular}{lrrrr}
\hline & Recarga & Transición & Descarga \\
\hline Máximo $\ldots \ldots \ldots \ldots \ldots \ldots \ldots$ & 11,20 & 8,37 & 6,40 \\
Media $\ldots \ldots \ldots \ldots \ldots \ldots \ldots$ & 5,22 & 2,00 & 1,52 \\
Mínimo $\ldots \ldots \ldots \ldots \ldots \ldots \ldots$ & $-0,71$ & $-5,00$ & $-6,74$ \\
\hline
\end{tabular}

Se aprecia claramente que el desequilibrio es mayor en las áreas de recarga y que, en líneas generales, el agua tiende a alcanzar el equilibrio entre la montmorillonita y la palygorskita hacia las áreas de descarga.

A modo de conclusión de este apartado, podemos afirmar que la extrapolación del modelo de transformaciones arcillosas sinsedimentarias propuesto por Trauth al sistema de flujo del agua subterránea, puede explicar ciertas anomalías geoquímicas observadas en dichas aguas. En el caso concreto del borde S.O. de la Cuenca del Duero, un paso concreto de la secuencia de Trauth, la transformación esmectita-palygorskita, no sólo es termodinámicamente posible, sino que además explica las pérdidas de sílice y $\mathbf{M g}^{++}$observadas en el sentido del flujo del agua subterránea. De acuerdo con Nesbbit (1985), este tipo de transformaciones o neoformaciones de minerales arcillosos debe, sin duda, explicar más fácilmente variaciones de la geoquímica de las aguas subterráneas atribuidas hasta ahora a supuestos intercambios catiónicos.

\section{Mineralogía y procesos de alteración en la formación areniscas de Aldearrubia}

Las Areniscas de Aldearrubia están constituídas por una asociación de cuarzo, micas individualizadas (moscovita y biotita), micas en agregados (micasquistos y clorosquistos) y feldespatos. Como accesorios aparecen apatito, circón, turmalina y trazas de cordierita. Una característica generalizada es la fuerte alteración que presentan las biotitas, cloritas y algunos feldespatos que se manifiesta por un reemplazamiento arcilloso más o menos importante; la moscovita y la microclina también presentan trazas de alteración pero en estados mucho menos avanzados.

La mineralogía descrita puede verse modificada mediante reemplazamiento epigenético por carbonato cálcico en los encostramientos de los fangos ocres bioturbados. Otra forma de acumulación de carbonatos es en cementaciones carbonatadas de origen diagenético más o menos tardío, localizadas en las facies arenosas más porosas.

La fracción inferior a $2 \mu$ está formada por illita, clorita, esmectitas y palygorskita, esta última en pequeña cantidad y muy variable. En la figura 2 
hemos representado un difractograma típico en la fracción $<2 \mu$ para cada una de las facies de la formación. En dicha figura se observa perfectamente cómo la cantidad de esmectita es inversa a la cantidad de clorita + illita. Este hecho está en concordancia con la alteración arcillosa observada en las cloritas, micas y algunos feldespatos. Así la alteración de cloritas y biotitas sería en forma de esmectitas, y, por tanto, a mayor alteración mayor cantidad de esmectitas. Como puede verse en la figura 2 , las mayores concentraciones de esmectitas, y, por tanto, los mayores procesos de alteración se localizan en las facies arenosas de depósito de canal y los menores en los fangos ocres de la llanura de inundación colonizadas por paleosuelos.

Recientemente ha sido propuesto un modelo de alteración que explica la alteración diferencial en las diversas facies de la formación (Alonso Gavilán et al., 1986). Unicamente cabe señalar aquí que dicho modelo sitúa la alteración en esmectitas del componentes arcósico de forma contemporánea con la sedimentación en estrecha relación con la dinámica del agua en la llanura de inundación.

Al otro componente de la arcilla - la palygorskitale dedicamos el apartado siguiente, no sin antes dejar constancia, como se ve en la figura 2 , que está presente en pequeña proporción y que su irregularidad de distribución, no permite correlacionarlo fácilmente con ningún otro fenómeno.

\section{La formación de la palygorskita}

La formación de palygorskita en la formación Areniscas de Aldearrubia puede atribuirse a los tres procesos siguientes: 1) Neoformación asociada a los encontramientos calcáreos producidos en los paleosuelos de los Fangos Ocres. 2) Formación asociada al proceso de alteración generalizado del componente arcósico de la formación y, por tanto, en paragénesis con las esmectitas. 3) Formación asociada a otro proceso de alteración diferente.

De las tres hipótesis la primera aparece, en principio, como la más plausible pues así ha sido clásicamente descrita en los procesos de encostramiento calcáreo de los suelos mediterráneos y subáridos (Millot et al., 1977). Un origen producido por este mecanismo necesitaría que las máximas concentraciones de palygorskita se diesen justamente ne los Fangos Ocres, ya que es en estas facies donde se ha producido los encontramientos calcáreos. Si bien, es efectivamente en esta facies donde hemos localizado algunos máximos de palygorskita, y, por tanto, no podemos excluir que una parte de este mineral tenga este origen, este mecanismo sólo no explica la presencia de palygorskita en las demás facies, ni su irregularidad de distribución.

La segunda hipótesis necesitaría una perfecta corre- lación esmectitas-palygorskita con identidad de máximos en las facies arenosas y de mínimos en los Fangos Ocres que está muy lejos de producirse.

Rechazadas al menos parcialmente las dos primeras hipótesis, es necesario definir un nuevo proceso que permita explicar la neoformación de la palygorskita. Un estudio realizado en microscopio electrónico de barrido en muestras representativas de las diversas facies de la formación ha aportado nuevos datos (lámina 1). En efecto, dicho estudio ha permitido observar cómo las fibras de palygorskita son posteriores a los procesos de alteración de la formación pues se observa perfectamente cómo las fibras envuelven los minerales alterados e incluso se desarrollan a partir de ella. Las fibras de palygorskita están asociadas, sobre todo, a los poros de las areniscas, que aparecen en general, tapizados por una fina malla de fibras. A partir de esta malla se inicia la construcción de una especie de «tela de araña" cuyo resultado final es el cierre del poro. Así han sido observados poros en todos los estadios del proceso, desde el inicio de la formación de una sola fibra hasta "telas de araña» muy complejas, que prácticamente han cerrado el poro inicial, pero que a su vez y sobre ella se crea una nueva microporosidad.

La presencia de palygorskita en cristales automorfos, en poros, no sólo se ha constatado en todas las facies de la formación, sino que además, en todos ellos se ha observado una gran irregularidad de distribución. Así aparecen de forma aleatoria, y a veces, estando contiguos poros casi «vacíos" y poros con «telas de araña» complejas. En cualquier caso, este modo de presentación de la palygorskita contrasta fuertemente con el de otras formaciones paleógenas o miocenas estudiadas por nosotros (Corochano et al., 1986; Armenteros et al., 1986), donde el origen del mineral está claramente asociado a los paleosuelos con encostramientos calcáreos. En efecto, en este caso las fibras de palygorskita aparecen organizadas en capas replegadas que contornean a los granos detríti$\cos$ o tapizan todo tipo de superficies edáficas: pedotúbulos, separaciones plásmicas, nódulos, etc.

Todas las características de la palygorskita de la formación Areniscas de Aldearrubia que acabamos de describir, y muy especialmente la presencia en cristales automorfos en los poros y el nulo control que ejerce la sedimentología sobre su distribución, creemos que aputan hacia un origen asociado a circulación de fluidos, afectando a toda la formación, y, por tanto, de origen diagenético tardío. El carácter «tardío» del proceso diagenético lo oponemos a los procesos de diagénesis «temprana» asociados a condiciones evaporíticas generalizadas o puntuales que han sido evocados en otros puntos, incluso de la Cuenca del Duero, como origen de la palygorskita y de la sepiolita (Galán y Castillo, 1984; Armenteros et al., op. cit.).

El contexto geológico de la Formación permite 


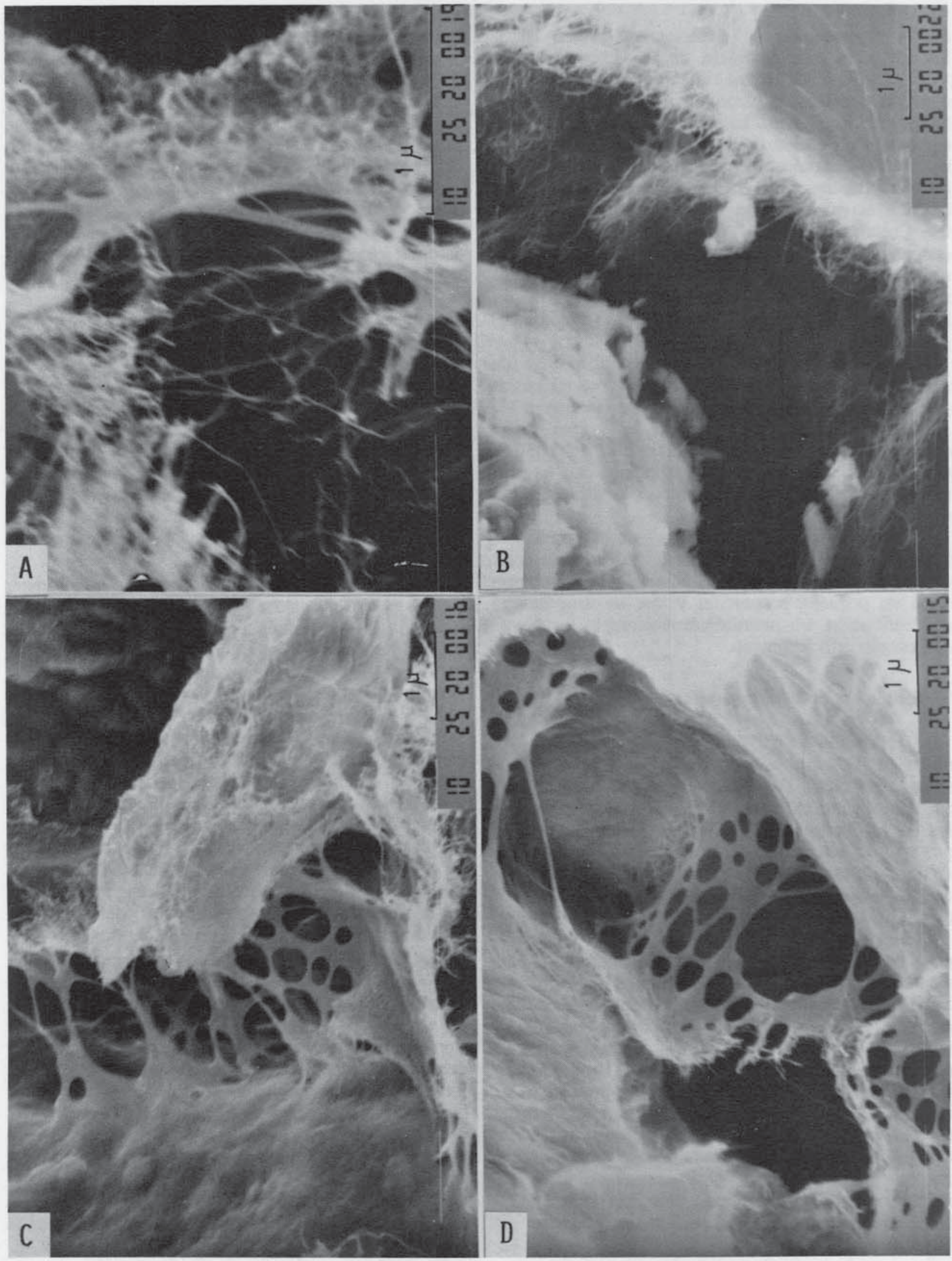

Lámina 1.-Diferentes aspectos al microscopio electrónico de barrido de la neoformación de palygorskita en la formación areniscas de Aldearrubia. Los primeros estadios de la neoformación, que se observan en las fotors A y B, se manifiestan por la aparición de un tapiz de fibras del mineral en el borde del poro. A partir de dicho tapiz, algunas fibras atraviesan completamente el poro y van a servir de base para la elaboración de complejas «telas de arañan que se aprecian en las fotos C y D. A pesar de todo, las «telas de araña» desarrollan una microporosidad que impide el cierre completo del poro. Por último, nótese la neoformación de fibras a partir de un mineral de tendencia hojosa (esmectitas?) en la foto C. 
hacer alguna precisión sobre las condiciones diagenéticas de formación de la palygorskita. En efecto, los afloramientos de la formación se sitúan en el borde W de la falla de Alba-Villoria, en el bloque levantado, donde queda excluído todo enterramiento considerable $\mathrm{y}$, por tanto, procesos diagenéticos que involucren un sustancial aumento de $P$ y $T$. Por otra parte, la pequeña cantidad de palygorskita presente en la formación y la irregular distribución a escala de cada poro, pensamos que indican mejor condiciones de formación relacionadas con el microsistema borde del poro-fluido, considerado cada poro aisladamente, que no un gran proceso que se pueda generalizar a toda la formación. Este mecanismo localizado en el micromedio definido por las paredes de un poro y el fluido circulante, creemos que explica razonablemente todas las observaciones realizadas sobre la palygorskita de la Formación Areniscas de Aldearrubia. La textura indica que su formación se produjo con posterioridad a todo tipo de procesos de compactación que de una u otra forma, hayan podido afectar a la porosidad de la Formación.

Resumiendo, hemos reducido para el proceso diagenético formador de la palygorskita las siguientes características: a) Carácter tardío en relación no sólo a la sedimentación, sino a todo tipo de procesos de compactación u otros que hayan podido actuar sobre la porosidad. b) Distribución aleatoria, en función de la dinámica del micro medio físico-químico definido por la pared de cada poro y el fluido circulante.

Un proceso diagenético, considerada la diagénesis en sentido amplio, que cumpla las características citadas, es la posible reacción de las aguas subterráneas con los materiales encajantes, lo que se conoce como "alteración de capa freática». Según este mecanismo de alteración, a mitad de camino entre las alteraciones de superficie y la diagénesis s.s., la palygorskita de la formación areniscas de Aldearrubia sería el resultado de la reacción entre ciertos minerales de la formación y las aguas subterráneas circulantes a través de ellos, actualmente o en el pasado.

A partir de las esmectitas de la formación, mediante reacción con sílice y $\mathbf{M g}^{++}$, que han podido ser aportados por las aguas subterráneas, no sólo es posible la elaboración de palygorskita sino que además dicha palygorskita debería situarse en la interfase agua-sedimento, es decir en la porosidad. Teniendo en cuenta que como hemos demostrado en la primera parte de este trabajo, las aguas subterráneas actuales pierden sílice y $\mathrm{Mg}^{++}$, nada nos impide pensar que la neoformación de palygorskita de la formación areniscas de Aldearrubia sea un proceso actual o subactual, por alteración de esmectitas.

Es evidente que los datos aportados en este trabajo son insuficientes, y en algunos casos cuestionables, para explicar y comprobar el proceso de palygorskiti- zación que se indica, pero aporta datos sobre la transformación esmectita-palygorskita, descrita por Weaver y Beck (1977) y aún no demostrada en laboratorio. Por otra parte, cuestiones como la posibilidad de formación de palygorskita a partir de otros minerales diferentes a las esmectitas, transformaciones previas de las propias esmectitas, en el sentido de hacerse cada vez más magnesianas, siguiendo el esquema de Trauth, y otras más, quedan por el momento sin respuesta.

\section{Conclusiones}

1) Las aguas subterráneas del borde SO de la Cuenca del Duero presentan a lo largo del flujo, desde las áreas de recarga a las áreas de descarga, una disminución de las concentraciones de silice y magnesio. Dicha disminución es más importante para la sílice, que en sus valores medios varía desde 32,4 ppm. en las áreas de recarga hasta $16,4 \mathrm{ppm}$. en las áreas de descarga; para el magnesio la variación va desde 26,7 hasta 14,2 ppm.

2) En una de las formaciones paleógenas de la zona -Areniscas de Aldearrubia - detectamos la formación en pequeñas cantidades de palygorskita que no es explicable por los procesos de alteración de la etapa sedimentaria o de la de diagénesis precoz. Dichos procesos de alteración se manifiestan por la alteración en esmectitas del componente arcósico de la formación que es muy regular en las distintas facies. La palygorskita, por el contrario, aparece de forma muy irregular pero en cristales automorfos aprovechando la porosidad.

3) La extrapolación al sistema «aguas subterráneasmateriales paleógenoss del modelo sinsedimentario de evolución de arcillas propuesto por Trauth (1977) puede justificar al mismo tiempo las observaciones 1 y 2. La extrapolación sería en el sentido: zona de borde $\sim$ zonas de recarga y zona centro $\sim$ zonas de descarga.

4) De esta secuencia el paso esmectita-palygorskita según Weaver y Beck (1977) debe realizarse siguiendo esta reacción:

Esmectita $+\mathrm{Mg}^{++}+\mathrm{SiO}_{4} \mathrm{H}_{4} \rightarrow$ Palygorskita $+\mathrm{H}^{+}+\mathrm{H}_{2} \mathrm{O}$

Lo que puede explicar la disminución de silice y $\mathrm{Mg}$ del agua con el aumento de palygorskita en los poros.

5) Este mecanismo de génesis de la palygorskita, a partir de "procesos de alteración de capa freática" aparece como una variante de los procesos diagenéticos tan frecuentemente invocados para la formación de palygorskita. 


\section{AGRADECIMIENTOS}

Este trabajo ha sido posible gracias a la colaboración de Philippe Larque y colaboradores, del Centre de Geochimie de la Surface de la Universidad Louis Pasteur (Stasbourg, Francia), de M. Sánchez Camazano y colaboradores, de la Unidad de Físico-Química de Arcillas del I.O.A.T.O. de Salamanca, y de Yves Noack y Michel Lanau del Laboratoire de Pétrographie te la Surface de la Universidad de Poitiers (Francia).

\section{Referencias}

Alonso Gavilán, G. (1981): Estratigrafía y sedimentología del Paleógeno en el borde Suroccidental de la Cuenca del Duero (provincia de Salamanca). Tesis Doctoral, Univ. Salamanca, $435 \mathrm{pp}$.

Alonso Gavilán, G. (1984): Evolución del sistema fluvial de la Formación Areniscas de Aldearrubia (Paleógeno Superior) (provincia de Salamanca). Mediterranea, 3: 107-130.

Alonso Gavilán, G. y Armenteros, I. (1983): Estructuras de deformación por escape de agua en los canales fluviales del Paleógeno Superior de Salamanca. Resúmenes y Comunicaciones. X Congr. Nac. Sedimentología. Menotca, 1983, 1: 21-22.

Alonso Gavilán, G.; Recio, C.; Fernández Macarro, B. y Blanco, J. A. (1986): Control sedimentológico de los procesos de alteración y rubefacción en la formación Areniscas de Aldearrubia (Eoceno Superior, Salamanca). Stvdia Geol. Salm., XXII: 37-59.

Armenteros I.; Fernández Macarro, B.; Recio, C. y Blanco, J. A. (1986): Análisis sedimentológico y paloegografía en el Mioceno del sector Sacramenia (Segovia)-Penafiel (Valladolid). Stvdia Geol. Salm., XXII: 247-262.

Corrochano, A. (1977): Estratigrafia y sedimentología del Paleógeno en la provincia de Zamora. Tesis Doctoral. Univ. Salamanca, $366 \mathrm{pp}$.

Corrochano, a. (1982): El Paleógeno del borde occidental de la Cuenca del Duero (provincia de Zamora). Temas Geológico Mineros, VI, I.G.M.E., Madrid, 687-700.

Corrochano, A.; Fernández Macarro, B.; Recio, C.; Blanco, J. A. y Valladares, I. (1986): Modelo sedimentario de los lagos neógenos de la cuenca del Duero (Sector Centro-Occidental). Stvdia Geol. Salm., XXII: 93-110.

Fernández, A. y Llamas, M. R. (1983): Avance sobre el estudio de la variación del contenido en sílice de las aguas subterráneas del sector oriental del Terciario detrítico del Tajo. III Simp. Nac. Hidrog., Madrid, VIII: 181-191.

Fritz, B. (1975): Etude thermodynamique et simulation des réactions entre mineraux et solutions. Aplication a la géochimie des alterations et des eaux continentales. Sci. Geol., Memorie 41, 153 pp., Strasbourg.

Galán, E. y Castillo, A. (1984): Sepiolite-Palygorskite in Spanish Tertiary Basins: genetical patterns in continental environments. In: Palygorskite-Sepiolite. Ocurrences, Genesis and Uses. (A. Singer y E. Galán, Eds.). Developments in Sedimentology, 37, Elsevier, 87-124.

Glass, H. D.; Potter, P. E. y Siever, G. (1956): The Clay Mineralogy of Some Basal Pennsylvanian Sandstones, Clays and Shales. Am. Assoc. Petroleum Geol. Bull., 40: 750-754.

Hubert, M. K. (1940): The Theory of Graoundwater Motion. J. Geol., 48: 785-944.
I.G.M.E. (1980 a): Mapa Geológico de España, Escala 1: 50.000, hoja núm. 397 (Zamora). Ministerio de Industria y Energía, Madrid.

I.G.M.E. (1980 b): Mapa Geológico de España, Escala 1: 50.000 , hoja núm. 426 (Fuentesauco). Ministerio de Industria y Energía, Madrid.

I.G.M.E. (1980 c): Investigación hidrogeológica de la Cuenca del Duero. Sistemas núm. 8 y 12 . Ministerio de Industria y Energía, Col. Informe, $75 \mathrm{pp}$.

I.G.M.E. (1982): Mapa Hidrogeológico de España, Escla I: 200.000, Hoja núm. 37 (Salamanca). Ministerio de Industria y Energía, Madrid.

Jiménez, E. (1972): El Paleógeno del borde Sur de la Cuenca del Duero. I: Los escarpes del Tormes. Stvdia Geol., 3: 67-110.

Jiménez, E. (1973): El Paleógeno del borde Sur de la Cuenca del Duero. II: La falla Alba-Villoria y sus implicaciones estratigráficas y geomorfológicas. Stvdia Geol., 5: 107-136.

Leguey, S.; Martín de Vidales, J. y Casas, J. (1984): Diagenetic Palygorskite in marginal continental detrital deposits located in the South of the Tertiary Duero Basin (Segovia, Spain). In: Palygorkite-Sepiolite. Ocurrences, Genesis and Uses (A. Singer y E. Galan, Eds.). Developments in Sedimentology, 37, Elsevier, 149-158.

Marti, B.; López, L. y Gómez, J. (1979): La utilización de los modelos matemáticos en la investigación de las grandes cuencas sedimenarias. Aplicación al estudio de la Cuenca del Duero. II Simp. Nac. Hidrog., Pamplona, V. 125-149.

Millot, G.; Nahon, D.; Paquet, H.; Ruellan, A. y Tardy, Y. (1977): L'épigenie calcaire des roches silicatées dans les encroutements carbonatés en pays subaride. Anti-Atlas, Maroc. Sci. Geol. Bull., 30: 129-152.

Nesbbit, H. W. (1985): A Chemical Equilibrium Model for the Illinois Basin Formation Waters. Amer. Jour. Science, 285: 436-458.

Potter, P. E. y Glass, H. D. (1958): Petrology and Sedimentation of the Pennsylvanian Sediments in Southern Illinois: A vertical Profile. Illinois Geol. Survey Rept. Inv., 204, 60 pp.

Sánchez, F. J. (1982): Hidrogeología del Terciario en el borde suroccidental de la Cuenca del Duero. Tesis Doctoral, Univ. Salamanca, $313 \mathrm{pp}$.

Sánchez, F. J. (1983): Hidroquímica del acuífero terciario al S.O. de la Cuenca del Duero. III Simp. Nac. Hidrog., Madrid, VIII, 137-146.

Tardy, Y. (1969): Géochimie des alterations. Etude des arenes et des eaux de quelques massifs cristallins d'Europe et d'Afrique. Mem. Serv. Carte Geol. Als. Lorr., 31, 199 pp., Strasbourg.

Tardy, Y. (1982): Kaolinite and Smectite Stability in Wathering Conditions. Estudios Geol., 38: 295-312.

Toth, J. (1963): A Theoretical Analysis of Groundwater Flow in Small Drainage Basins. J. Geophys. Res., 68: 4795-4812.

Trauth, N. (1977): Argiles evaporitiques dans la sedimentation continentale et epicontinentale tertiaire. Basin de Paris, de Mormoiron et de Salinelles (France). Sci. Geol., Memoire 49, 195 pp., Strasbourg.

Weaver, C. E. y Beck, K. C. (1977): Miocene of the S.E. United States. A Model for Chemical Sedimentation in a Perimarine Environment. Developments in Sedimentology, 22, Elsevier, 234 pp.

Recibido el 21 de enero de 1986 Aceptado el 16 de octubre de 1986 